\title{
EL CAPITAL INTELECTUAL EN UN HOSPITAL DE ALTA ESPECIALIDAD MATERNO PERINATAL. CASO DE ESTUDIO
}

\author{
Intellectual capital in a pernital maternity \\ hospital of high speciality. Case study
}

\section{Patricia Mercado-Salgado ${ }^{1}$}

Fecha de recepción: 27 de febrero del 2018

Fecha de aceptación: 19 de marzo del 2018 


\section{Resumen}

El desempeño de las organizaciones de salud está parcialmente sustentado en los stocks de capital intelectual. Aunque el sector salud es intensivo en conocimiento, distribuido en la mente de los individuos, capturado en los expedientes, en bases de datos y en reportes, e incrustado en comités y rutinas organizacionales, son insuficientes los estudios que exploran la presencia del capital intelectual como recurso intangible para un desempeño superior. El objetivo de este caso de estudio es identificar las manifestaciones del capital intelectual (capital humano, capital estructural y capital relacional) en un hospital materno perinatal para mejorar su aprovechamiento. La relevancia de los resultados está en manifestar que el capital intelectual por sí sólo no logra su cometido; debe vincularse a los recursos tangibles (por ejemplo, medicamentos e instalaciones), para construir la dinámica cotidiana que demanda la reducción de la mortalidad materna en mujeres del Estado de México.

Palabras clave: capital intelectual, capital humano, capital estructural, capital relacional, hospital público.

\section{Abstract}

The performance of health organizations is partially supported by the stocks of intellectual capital. Although the health sector is knowledge-intensive, distributed in the minds of individuals, captured in files, databases and reports, and embedded in organizational routines and committees, there are insufficient studies to explore intellectual capital as a iintangible resource for superior performance. The objective of this case study is identify intellectual capital manifestations (human capital, structural capital and relational capital) in a maternal perinatal hospital to improve its use. All alone intellectual capital does not achieve its purpose. It must be linked to tangible resources (for example, medicines and facilities), to build the daily dynamics required in reduction maternal mortality in women from State of Mexico.

Keywords: Intellectual capital, human capital, structural capital, relational capital, public hospital. 


\section{Introducción}

$\mathrm{P}$ ara mejorar la calidad de atención, contener los crecientes costos e integrar los servicios de salud, se necesita mayor eficiencia en la gestión de recursos tangibles e intangibles, tanto a nivel intra como inter organizacional.

Sobre los recursos intangibles, hace ya 15 años Ley y Zambon (2003) argumentaban la demanda de más investigación, específicamente sobre capital intelectual como conductor del crecimiento organizacional en una economía donde la innovación, las tecnologías de información y comunicación, las redes y las alianzas, así como la calidad y organización de los recursos humanos, resultan vitales para la competitividad de las organizaciones. Desde entonces, y aún antes, algunas dificultades para el estudio del capital intelectual han estado enraizadas en su naturaleza multiforme y el poco consenso en su definición, medición y gestión.

Coincidente con lo anterior, Evans, Brown y Baker (2015) confirman, mediante una revisión sistemática y crítica de la literatura sobre capital intelectual en organizaciones de salud, que su desempeño superior se deriva de una combinación compleja de recursos tangibles e intangibles. En nuestra sociedad basada en el conocimiento, los intangibles y no los tangibles, favorecen más el cumplimiento de objetivos $y$, por ende, la atención de apremiantes necesidades de salud en población vulnerable.

$\mathrm{Al}$ respecto, este caso de estudio con alcance exploratorio y descriptivo, aborda el capital intelectual conjuntamente con la disponibilidad de recursos tangibles. El objetivo es identificar las manifestaciones de capital intelectual (capital humano, capital estructural y capital relacional), tomando como laboratorio de estudio un hospital materno perinatal de atención a la población abierta ubicado en el Estado de México.

Con esto se contribuye a apoyar la decisión de gestionar el capital intelectual y, al mismo tiempo, generar la oportunidad de entrelazar la producción de la comunidad académica con el quehacer cotidiano de los tomadores de decisiones en unidades de salud.

Para cumplir con lo anterior, este documento se conforma de cuatro secciones. En la primera se presenta una reflexión sobre los retos que enfrenta la investigación del capital intelectual, tomando como eje la Teoría de Recursos. La siguiente sección contiene el contexto del hospital en cuestión, justificando la importancia de su labor desde la reducción de la mortalidad materna en población económicamente vulnerable. Posteriormente, se presentan las características metodológicas de este caso de estudio, para cerrar con los resultados (cuarta sección) conformados en dos partes. Primero, las manifestaciones de interrelación entre capital humano, capital estructural y capital relacional; segundo, la co-dependencia del capital intelectual y los recursos tangibles en su contribución en el desempeño superior de un hospital de alta especialidad materno perinatal que brinda atención a la población abierta, es decir, a mujeres económica y socialmente vulnerables.

\section{El enfoque de Recursos en la investigación del capital intelectual en organizaciones de salud}

Evans et al. (2015) confirman, mediante una revisión sistemática y crítica, que la publicación de literatura científica sobre capital intelectual en organizaciones de salud va en aumento, sin que el conocimiento avance. Las limitaciones teóricas y metodológicas siguen latentes y la aplicación práctica en las instituciones dedicadas al cuidado de la salud humana es limitada. 
Si bien el concepto, la estructura tripartita (capital humano, capital estructural y capital relacional) y la medición cuantitativa del capital intelectual han servido de marco para estudiar su potencial en el desempeño organizacional, sigue dejándose de lado la relación sinérgica que detonan los recursos tangibles en este tipo de instituciones, donde medicamentos, material de curación, instalaciones equipadas e insumos (como oxígeno e instrumental quirúrgico) son imprescindibles e insustituibles.

Al respecto, Kong (2008) y Kong y Prior (2008) proponen que los recursos tangibles e intangibles están anidados con interrelaciones y dinámicas específicas. Para Peng et al., (2006), una organización puede adquirir sus recursos esenciales dentro y fuera de sus límites, pues no sólo se construyen internamente, sino también se obtienen mediante alianzas o redes.

Barney (1991) define los recursos como atributos que pueden permitir a las empresas concebir e implementar estrategias de creación de valor y desempeño superior frente a la competencia o sus similares. Incluyen cualquier cosa (activos, capacidades, procesos, atributos gerenciales, información, conocimiento, capital físico y capital humano, por ejemplo) que pueda ser controlada y utilizada por la empresa para planear e implementar estrategias que mejoren su eficiencia y efectividad.

Desde la Teoría de Recursos, algunos tangibles e intangibles son más valorados que otros, dependiendo de qué tan valiosos, escasos, difíciles de imitar e insustituibles lleguen a ser para el desarrollo de las actividades productivas y la creación de valor (Jang, 2013). Cada organización tiene su propia estrategia para allegarse y disponer de recursos de manera eficiente. La mayoría de las veces, dicha estrategia es producto de su historia, decisiones pasadas y contexto (Barney, 1991; Grant, 1991).

Este enfoque teórico argumenta que las diferencias en el desempeño entre organizaciones, son resultado de la heterogeneidad de los recursos tangibles e intangibles, así como la manera en que ambos se integran. La teoría basada en el conocimiento es una extensión de la teoría de recursos que enfatiza el conocimiento y el aprendizaje como recursos intangibles críticos, e identifica la razón fundamental de la empresa como la creación y aplicación de conocimiento (Bontis, 1999).

El capital intelectual como recurso, es la suma de stocks de conocimiento que las organizaciones usan para crear valor, ventaja competitiva y diferenciación entre organizaciones similares del mismo sector con ubicación territorial compartida. En el contexto de la gran empresa capitalista, Stewart (1997) especifica que dicho conocimiento debe estar formalizado, capturado y apalancado financieramente para producir riqueza. Para Bradley (1997) el capital intelectual es la habilidad para transformar conocimiento y activos intangibles en recursos de creación de riqueza, tanto para las organizaciones como para las naciones.

El capital intelectual en las organizaciones es acumulado y distribuido en tres diferentes vías: a través de los individuos (capital humano), mediante las estructuras, procesos y sistemas de la organización (capital estructural) y por las relaciones y redes (capital relacional) (Ahmad, Naji y Bontis, 2010). El capital humano se relaciona con todos los recursos incorporados en el sujeto empleado por la organización. El capital estructural se refiere a los recursos que la organización ha desarrollado o producido y que posee legalmente, tales como marca, procesos, rutinas, sistemas, estructuras e información en bases de datos o en papel. Los recursos del capital relacional abarcan las relaciones que la organización tiene al interior y con entidades externas, pero que influyen en su capacidad de la organización para crear valor (Roos et al., 2005).

Para justificar por qué las organizaciones deberían desarrollar mayor interés en el capital intelectual, Dumay (2012) recurre a dos enfoques: (a) la diferencia entre el valor de mercado y el valor de libros; (b) un mayor rendimiento mediante la detección y gestión de este intangible. El argumento del primero es 
que la valoración de mercado de algunas empresas es mayor que sus activos netos informados, los cuales pudieran estar subestimados. Si bien la noción anterior es lógica, en la práctica no parece haber logrado una amplia aceptación en la comunidad empresarial, como lo demuestra la falta de proliferación de las empresas que informan externamente sobre su capital intelectual, ya sea por sesgos en la información o por no tener claro los vínculos causales entre los intangibles específicos y la creación de valor.

Al respecto, Booker, Bontis y Serenko (2008) afirman que el campo de estudio del capital intelectual es atractivo, tanto para profesionales como para académicos, pero también se cuestionan si en realidad está siendo útil, ya sea para la formación de futuros gerentes o para la generación de conocimiento aplicable en la cotidianidad de las organizaciones, ya que existen publicaciones que generan prescripciones, hacen recomendaciones, ofrecen soluciones y desarrollan principios aplicables a la práctica gerencial, pero poco recurren a ello los tomadores de decisiones.

Bontis, Crossan y Hulland (2002) argumentan que la aplicación de prácticas de gestión de capital intelectual tiene un impacto positivo en las capacidades de aprendizaje organizacional. Sin embargo, la débil claridad conceptual del capital intelectual y la confusión que se genera con ello, pudieran estar dando lugar a que la mayoría de las organizaciones de salud no cuenten con un enfoque integral y sistemático para gestionar el capital intelectual, sin hacer de lado el poco interés por parte de los directivos (Evans, et al 2015).

Ante las oportunidades para mejorar el vínculo entre el trabajo de los académicos y los tomadores de decisiones en las organizaciones, es crucial generar resultados de investigación que estén alineados para contribuir a los retos que enfrentan las instituciones de salud.

Con el presente estudio se busca, entonces, contribuir a disminuir la brecha entre lo abstracto del capital intelectual y las aplicaciones prácticas, mediante la detección de algunas manifestaciones de capital humano, capital estructural y capital relacional en un hospital de alta especialidad materno perinatal, como laboratorio de este caso de estudio.

Aunque el concepto y estructura del capital intelectual ofrecen un medio para estudiar su valor y potencial en el desempeño organizacional, todavía reta a investigadores y tomadores de decisiones a identificar y mejorar estos recursos intangibles y determinar cómo generar sinergias con los recursos tangibles, pues lo que en una industria u organización es capital intelectual, pudiera no serlo en otra, debido a diferencias en las estrategias y el ambiente. De aquí que para desempacar y explorar el capital intelectual en un hospital de atención materno perinatal, es necesario conocer algo de su complejo contexto.

\section{El contexto: del embarazo de alto riesgo al hospital materno perinatal de alta especialidad}

El embarazo es un periodo que puede representar una sobrecarga significativa al cuerpo de la mujer, ya que no hay un solo órgano que no presente modificaciones biológicas durante el proceso de gestación. El embarazo de alto riesgo se caracteriza porque la mujer presenta complicaciones y requiere de atención clínica especializada para evitar la mortalidad materna (Karam et al., 2007).

Se estima que alrededor de $20 \%$ de los embarazos son de alto riesgo y responsables de más de $80 \%$ de los resultados perinatales adversos, tales como la prematura, la asfixia perinatal, las malformaciones congénitas y las infecciones. Desde el punto de vista materno están la hemorragia obstétrica, las infecciones, el 
síndrome hipertensivo del embarazo y las enfermedades pregestacionales, mismos que demandan estrategias de prevención, diagnóstico y tratamiento oportunos (Donoso y Oyarzún, 2012).

De los embarazos de alto riesgo reportados en México, aproximadamente 20\% corresponde a casos en el Estado de México, lo que equivale a que cada año se registran alrededor de 9,500 embarazos en la entidad mexiquense, cifra total que representa el índice de cinco países europeos en conjunto. En esta entidad, alrededor de $50 \%$ de las mujeres que presentan este tipo de embarazo muere a causa de una hemorragia cerebral derivada de la hipertensión grave o preclamsia, enfermedad que se posiciona como la primera causa de muerte por embarazo de alto riesgo (ISEM, 2017).

Aunque existe una gran discrepancia en las estadísticas de mortalidad materno perinatal entre los países subdesarrollados, en América Latina se le relaciona con barreras a la educación en las mujeres, la pobreza y la elevada fertilidad (Aristizábal et al., 2005).

La mortalidad materna es considerada un indicador sensible al desarrollo social, pues la pérdida inesperada de la madre genera consecuencias emocionales, económicas y de salud en el núcleo familiar, por lo que esta problemática debe ser abordada de manera integral con el fin de mitigar impactos negativos (Lozano, Bohórquez y Zambrano, 2016).

En este mismo sentido y en el contexto mexicano, las severas consecuencias futuras para los niños que se quedan sin madre se traducen en morbimortalidad infantil, deserción escolar e incremento de violencia y delincuencia, entre otros problemas sociales. También es un problema de salud pública, pues refleja las deficiencias en el acceso y la calidad de los servicios de atención a la salud materna que son ofrecidos a la población.

En esencia, reducir las muertes maternas requiere de acciones coordinadas y de un trabajo conjunto, desde el interior de las familias hasta las políticas nacionales (Karam et al., 2007).

Ante este escenario y en el marco de las metas de los Objetivos de Desarrollo Sostenible y la Estrategia Mundial para la Salud de la Mujer, el Niño y el Adolescente emitidas por la Organización Mundial de la Salud, México ha establecido políticas y acciones para detener las complicaciones maternas.

Específicamente, el Estado de México tiene un modelo de atención que prioriza las necesidades de grupos vulnerables. Tal es el caso de este centro hospitalario de atención especializada en el área crítica materna perinatal que atiende a población abierta desde 2009 para brindar servicio a mujeres que presentan embarazos de alto riesgo y a niños recién nacidos en condiciones críticas.

En cuanto a su infraestructura y tecnología, este nosocomio cuenta con 19 consultorios, tres quirófanos (uno de alta especialidad único en el país) y dos salas de expulsión. Brinda estancia hospitalaria de tipo obstétrica, neonatal, medicina aguda y ginecológica, diseñada para la paciente altamente complicada y equipada con tecnología de punta para la detección, tratamiento y vigilancia de padecimientos relacionados con la salud de la mujer y del neonato. Alberga especialidades de anestesiología, cardiología, cardiología pediátrica, cirugía general, clínica de la fertilidad, clínica de mama, epidemiología, estimulación temprana, genética, ginecología, medicina critica, medicina interna, medicina materno-fetal, neonatología, neurocirugía, neurología, nutrición, obstetricia de alto riesgo, pediatría, planificación familiar, psicología, psiquiatría, traumatología y ortopedia, urgencias y urología ginecológica. Está certificado como Hospital Amigo del Niño y de la Niña, iniciativa mundial lanzada por la Organización Mundial de la Salud (OMS) y el Fondo de las Naciones Unidas para la Infancia (UNICEF) con el propósito de implementar prácticas que protejan, promuevan y apoyen la lactancia materna. 
La misión de este hospital es proporcionar servicios de salud materno neonatal por personal altamente especializado, sustentados en la aplicación de tecnología de vanguardia, dentro de un marco ético y humanístico. Esta organización de salud busca el reconocimiento social por su compromiso con el servicio, su modelo de atención, la formación de especialistas y el desarrollo de investigación científica materno neonatal.

Para reducir la morbi-mortalidad de una población altamente vulnerable como lo es la mujer embarazada y los recién nacidos ( 0 a 29 días) en condiciones críticas, todavía debe enfrentar el reto impostergable de la transformación de la cultura organizacional hacia nuevos esquemas de responsabilidad, transparencia, organización, liderazgo y productividad (ISEM, 2017). Una alternativa viable para ello, es el aprovechamiento de sus recursos tangibles e intangibles, especialmente los englobados en el capital intelectual.

\section{Método}

Un punto de partida de este estudio fueron algunas controversias en la medición cuantitativa del capital intelectual en hospitales. En otras palabras, no se utilizó un cuestionario centrado en la percepción del personal sobre el capital intelectual, pues hay reactivos tan generales que bien pueden atrapar un constructo diferente. Ejemplo de ello es: "el personal de esta organización cuenta con las capacidades requeridas para desempeñar su trabajo" (Evans et al., 2015; Kannan y Aulber, 2004).

Se recurrió a un diseño de investigación cualitativo y como método al caso de estudio. Desde el enfoque cualitativo se construyó la detección del capital intelectual en un hospital de alta especialidad para el cuidado de la embarazada y el neonato en alto riesgo, tomando en cuenta el punto de vista de 20 participantes (médicos y enfermeras), documentos institucionales (planes y programas) y una entrevista a profundidad de un alto directivo.

Para alejarnos de la atinada crítica de Evans et al. (2015), las aportaciones de los participantes se recopilaron mediante un ejercicio escrito donde se les solicitó ejemplificar el capital humano, el capital estructural y el capital relacional en su ambiente de trabajo, así como las amenazas que enfrentan y la manera en que pueden ser mejor aprovechados. Este ejercicio estuvo precedido por la ponencia "Gestión del capital intelectual en instituciones de salud", impartida en el marco de la Jornada de Capacitación 2016 organizada por el propio hospital.

Lo anterior queda enmarcado en el caso de estudio como una estrategia de investigación que permite la exploración a profundidad de un evento, limitado en un tiempo y actividad específicos, para lo cual puede recurrirse a una diversidad de procedimientos y fuentes para la obtención de datos e información (Creswell, 2009; Remenyie, 2012; Yin, 1989).

Una ventaja de esto es ofrecer a los directivos del nosocomio una narrativa del status del capital intelectual, desde dos aristas: la interrelación entre el capital humano, el capital estructural y el capital relacional, y la manera en que los intangibles están incrustados con los tangibles. Si bien éstos son más visibles, la combinación única que pueda hacer la organización, es lo que le da el valor estratégico para cumplir con sus objetivos y metas. 
La estructura de la narrativa presentada en los resultados se construyó a partir de dos bloques: la interrelación entre los componentes del modelo tripartita del capital intelectual y la co dependencia de los intangibles con los tangibles.

Las categorías asociadas a cada código se detectaron una vez transcritas las respuestas de los participantes y la entrevista a profundidad, lo cual se reforzó con los documentos institucionales disponibles. Dichas categorías son capacitación, equipo de trabajo, liderazgo, actitudes y valores para el capital humano; comités y normatividad para el capital estructural y el capital relacional se conformó por las redes de hospitales, las relaciones paciente-familiares-personal de salud, colaboración de equipos multidisciplinarios, trabajo social y comunidad virtual. En la interacción de intangibles con tangibles predominan las tecnologías de información y comunicación.

Vale la pena mencionar que no se recurrió al cálculo de frecuencias por dos razones. Primera, se detectó la interconexión entre los tres capitales (humano, estructural y relacional), y no el de mayor relevancia. Segunda, la narrativa es a partir de las actividades/tareas/objetivos del hospital y cómo se insertan estos capitales en ellas.

Para resaltar la interrelación entre los componentes del modelo tripartita del capital intelectual, a lo largo del texto se anota entre paréntesis el código de referencia: $\mathrm{CH}$ para capital humano, CE para capital estructural y CR para capital relacional.

\section{Resultados}

Para presentar las manifestaciones, expresiones o ejemplos de capital intelectual en un hospital materno perinatal de alta especialidad que brinda atención a la población abierta, contexto de este caso de estudio, se acotó la diversidad de propuestas existentes en la literatura a partir del modelo tripartita (capital humano, capital estructural y capital relacional), tomando en cuenta los dos criterios de Evans et al. (2015). Primero, para generar y mantener el conocimiento que contribuya a resultados superiores de la organización, los componentes del capital intelectual son interdependientes y se activan colectivamente al conformar el stock de conocimiento, aunque en las publicaciones científicas es frecuente que se reporte el capital humano, el capital estructural y el capital relacional como constructos separados con sus antecedentes y consecuentes, lo que tal vez, acentúe la falta de interés por este intangible en quienes toman las decisiones. Segundo, el capital intelectual actúa, desde la Teoría de Recursos, en vínculo con los recursos tangibles para influir en los resultados de desempeño superior de las organizaciones de salud.

\subsection{Interrelación entre capital humano, capital estructural y capital relacional}

En el contexto de las organizaciones de salud, marco del capital intelectual, el capital humano son conocimientos, habilidades y experiencias que posee y usa el personal para brindar atención de calidad y con calidez a los pacientes. En el cuidado especializado de enfermos de alto riesgo materno perinatal, el capital humano son competencias profesionales (duras y blandas), habilidades de gestión y liderazgo, actitudes y conocimiento específico, lo cual corresponde a las 21 especialidades con que cuenta este 
hospital de tercer nivel, así como a los médicos y paramédicos altamente capacitados en clínica de la fertilidad, lactancia materna, neonatología, clínica de catéteres y dominio de las nuevas técnicas para la contención de la hemorragia post-parto y el uso del cool-cap (estrategia beneficiosa) en la encefalopatía hipóxica isquémica del recién nacido.

Aunque no siempre puede evaluarse si los recursos humanos, las competencias y los procesos están en el lugar preciso para contribuir al desarrollo exitoso, al cambio o a la innovación (Kannan y Aulber, 2004), el capital humano en este nosocomio se nutre con la formación continua, ya sea con capacitación formal presencial y a distancia, o con la práctica de acompañamiento in situ.

La Unidad de Enseñanza e Investigación, en acompañamiento con el Comité de Enseñanza Médica del hospital, es la responsable de planear, coordinar y supervisar programas y acciones orientados a la formación, capacitación y desarrollo de recursos humanos especializados en la atención de la salud materno perinatal. Promueve la formación de capital humano mediante cursos de pregrado (licenciaturas, tesis y servicio social) y postgrado (especialidades, maestrías y doctorados).

La capacitación en el aspecto crítico de la atención perinatal se lleva a cabo con actividades internas (socialización del conocimiento) y externas vinculadas a la difusión del conocimiento (CR), con soporte de tecnologías de información y comunicación (capacitación a distancia).

Para mantener actualizados los conocimientos específicos, el personal de enfermaría también recurre a la capacitación continua (diplomados y cursos a nacional e internacional) y participa en actividades de enseñanza e investigación en materia de enfermería quirúrgica y perinatal.

Este hospital, con aval de la Facultad de Medicina de la Universidad Autónoma del Estado de México (CR), ocupa un lugar estratégico en la formación de médicos de alta calidad profesional y humanística en la especialidad de ginecología y obstetricia y en las subespecialidades de neonatología y medicina crítica en obstetricia.

De manera paralela a la capacitación, se ejecutan acciones para la generación de investigación en materia de salud materno perinatal, así como su difusión y aplicación en el área médica y de enfermería. Para ello, se supervisa el desarrollo de los proyectos de investigación (internos y externos), a efecto de evaluar sus resultados y alcance con rigor técnico-científico, así como los beneficios socio-económicos en las áreas clínicas, biomédicas, socio-médica y otras vinculadas con el quehacer del hospital (CR). Mediante la publicación de artículos científicos de autoría del personal especializado, el hospital se está proyectando a nivel nacional e internacional (CR). Específicamente, el Banco de Sangre promueve la investigación y capacitación en medicina transfusional.

Otra manifestación del capital humano es el equipo médico de emergencias, conocido como Código Mater, Emergencia Obstétrica o Equipo de Respuesta Inmediata Obstétrica, para el manejo del paciente de embarazo de alto riesgo y su atención en el cuidado intensivo, el cual surge como estrategia para reducir la razón de mortalidad materna. Este equipo de expertos, y resolutivo en casos de extrema gravedad, evalúa y estabiliza la condición del paciente e interviene en su tratamiento y cuidado. Su objetivo es responder ante el primer dato de alarma, de acuerdo al Manual de Procedimientos de Triage y a la Tabla de Priorización para la Atención de la Mujer Embarazada (CE), antes de que se convierta en una serie de complicaciones orientadas a la catástrofe del tratamiento.

Dicho equipo está conformado por especialistas en ginecología y obstetricia, cuidados intensivos, anestesiología, cirugía general y neonatología, dos enfermeras y un camillero, acompañados de servicios 
de laboratorio, banco de sangre, imagenología, trabajo social y responsable de las áreas administrativas. Este equipo médico de emergencia también participa en la capacitación in situ de médicos y enfermeras, en base a los hallazgos clínicos acerca de la estructuración, acciones, responsabilidades y áreas de oportunidad que en la práctica se presentan. Para mantener la certificación (CE) del Código Mater, es ineludible que en cada turno y por servicio se cuente con una enfermera capacitada y actualizada en reanimación neonatal, rubro hasta ahora pendiente.

Las habilidades de liderazgo son el eje para gestionar la dinámica humana, tomando en cuenta las competencias, las actitudes y la agilidad mental del personal de salud en una mezcla de educación formal e informal. En este sentido, además de la toma de decisiones asertiva y oportuna, es tarea de la alta gerencia y los mandos medios mantener un equipo de trabajo comprometido con los fines institucionales. Sin embargo, el personal de este hospital percibe cierta falta de comunicación efectiva y algunas oportunidades de mejora en la organización de tareas y en la distribución de recursos financieros y materiales.

En concordancia, la importancia de las actitudes en el concierto del capital humano, radica en que ayudan a predecir el comportamiento hacia el trabajo, a adaptarnos al ambiente laboral y a disminuir la resistencia al cambio (Luthans, 1989). Específicamente, el personal de salud en este nosocomio debe ser alertado para que la antigüedad laboral no de paso al desinterés, a la apatía y al cansancio emocional, ni mucho menos ataque a la innovación organizativa. Por ejemplo, se detecta cierta apatía hacia las sesiones generales de capacitación con periodicidad semanal, aunque en contraste, se demanden mayores oportunidades para mantener la requerida actualización. La misma suerte corre el Programa Específico de Capacitación, el cual es de carácter obligatorio con calendarización al inicio de año. Parece, entonces, que la falta de actitud hacia el aprendizaje es una causa de la inasistencia a las conferencias impartidas por especialistas invitados.

El capital humano se refiere a quienes mediante competencias valiosas y distintivas contribuyen a generar valor para el hospital, pero también para ellos mismos. Al ser una alternativa la inversión intangible a nivel personal, grupal y organizacional, el capital humano reclama una permanente atención en corresponsabilidad, reflejada en la filosofía que las organizaciones tengan de él. Tal es el caso del hospital materno perinatal, cuando incluye en su misión y visión "la formación de recursos humanos de excelencia".

El personal hospitalario reconoce como valores guía la responsabilidad, el conocimiento especializado y la calidez humana en la atención del paciente. A nivel organizacional, la bioética hospitalaria es el eje transversal de la actuación del personal de salud y administrativo, puesto que la medicina combina en su propia naturaleza un quehacer moral y uno técnico científico, dotado de gran carga humanística, a fin de respetar la integridad personal. Este hospital cuenta con el Comité de Ética en Investigación, el Comité de Investigación y el Comité Hospitalario de Bioética, buscando la generación de investigación de calidad en apego al cuidado de los aspectos técnicos, científicos y sobre todo humanos (CH y $\mathrm{CE}$ ). Estos comités están constituidos para deliberar sobre la moralidad de determinadas decisiones o cursos de acción, bien sea en la práctica clínica, en la investigación científica o en la gestión de los recursos sanitarios disponibles.

La teoría de ajuste persona-ambiente asume que los individuos prefieren un lugar de trabajo con características similares a ellos (por ejemplo, valores y creencias). En este sentido, cuando el personal "se ajusta" con la organización, el nivel de congruencia entre valores de los empleados y el hospital marca mayor satisfacción y compromiso, como actitudes en el ambiente laboral (Amos y Weathington, 2008). 
Es decir, cuando existe congruencia entre los valores de un empleado, los de la organización y los de los colegas, las actitudes hacia el trabajo (satisfacción y compromiso) tienden a ser positivas (Tzu-Ju, 2011).

Puesto que la vasta existencia de know-how (formal e informal) está distribuido en la mente del personal $(\mathrm{CH})$, el conocimiento organizacional puede quedar capturado en expedientes clínicos, bases de datos y reportes para conformar así el capital estructural e incrustarse en la cultura y rutinas organizaciones, mediante planes estratégicos, programas, sistemas de información, mejores prácticas y rutinas.

Este hospital se caracteriza como innovador en su organización, formulación y aplicación de procesos, procuración de servicios de salud de la paciente obstétrica crítica, conformación de equipos de trabajo de respuesta inmediata y guías de práctica clínica actualizadas. Para ello, y como expresión de un arduo trabajo colaborativo $(\mathrm{CH})$, en este nosocomio existen comités e indicadores que dan cuenta de los avances en el combate a la muerte materna.

Además de los ya referidos comités de enseñanza, investigación y ética, la organización del hospital está sustentada en otros más: el Comité de Calidad y Seguridad del Paciente (COCASEP), encargado de sesionar sobre casos que han trascendido a la muerte o una secuela grave y permanente en el paciente; el Comité de Detección y Control de Infecciones Nosocomiales (CODECIN); el Comité de Farmacia y Terapéutica (COFAT) acompañado de tres sub comités (fármaco-vigilancia; calidad y seguridad de la atención médicaexpediente clínico; prevención, estudio y seguimiento de la morbilidad y mortalidad materna y perinatal hospitalaria); el Comité de Cesáreas, así como los sub comités de Lactancia Materna, Biología de la Reproducción y Medicina Transfusional. Las sesiones mensuales de estos comités se calendarizan a inicios de cada año.

Este modo de organización puede ser considerado una manifestación de capital estructural, toda vez que el establecimiento de diversos comités se basa en la respectiva legislación y su existencia se justifica al abordar problemas a través de personal especializado y empoderado, lo que marca su alcance, pues quienes están al frente de cada comité son especialistas y ostentan jefaturas o subdirecciones. Mediante esta figura de comités se hacen cumplir las normas y son una piedra angular de las políticas de salud.

La manera en que los comités están estructurados y cómo funcionan $(\mathrm{CH})$, hacen la diferencia en los resultados. En cuanto a su estructura, Eaton y Nocerino (2000) detectan factores internos y factores externos (o contextuales). En los primeros están la periodicidad y regularidad de las reuniones, la comunicación con personal y directivos que no pertenecen al comité, el cumplimiento a la agenda de trabajo, la elaboración de actas, la capacitación como miembros del comité y la percepción de su auto eficiencia. Cuando la alta gerencia está involucrada $(\mathrm{CH})$, el comité percibe ser más eficiente, pues el compromiso directivo es importante para lograr el cometido de estas figuras colectivas internas.

Si bien lo más importante en la efectividad de los comités son sus factores internos, la presión gubernamental como factor externo llega a enturbiar el trabajo de los comités, sobre todo cuando las recomendaciones emitidas tienden a trastocar intereses de terceros involucrados con posturas privilegiadas. Sin embargo, la presión que el gobierno tiene por parte de una sociedad cada vez más informada, impulsa a los comités y a la gerencia hospitalaria a trabajar conjuntamente $(\mathrm{CH})$.

En otras palabras, las medidas de presión externa tienen un efecto significativo (positivo o negativo) en los resultados del comité. Lo importante es evitar la confusión entre medios y fines (o resultados) de los comités, y para ello, su efectividad debe medirse por el impacto en la disminución de muertes maternas, así como por el número y pertinencia de las recomendaciones emitidas a partir de la identificación de causas raíz (CR). 
Ejemplo de efectividad de los comités en este nosocomio es la aplicación del Diagrama de Ishikawa, herramienta que representa la relación entre un efecto (problema) y todas las posibles causas que lo ocasionan, para analizar los eventos adversos a partir de hechos, incluyendo el turno, el paciente y los responsables de las fallas, con la finalidad de detectar la ruptura de la cadena de seguridad de atención al paciente ( $\mathrm{CH}$ y CE).

Entre la normatividad del hospital (CE) está el plan de trabajo anual, en el cual se incluyen indicadores de resultados (consultas de especialidad, atenciones de urgencias, nacidos vivos, defunciones, estudios de laboratorio y tamiz neonatal) en comparativos con los últimos cinco años, de donde se desprende un análisis de fortalezas-oportunidades y debilidades-amenazas (FODA), así como estrategias de mejora para la disminución de la muerte materna y neonatal. Este plan de trabajo tiene como objetivo establecer, coordinar y supervisar el ejercicio de los programas para la atención asistencial de mujeres con embarazo de alto riesgo, problemas de patología ginecológica, gineco-oncológica, uroginecológica, reproductiva y neonatal, a través de la correcta aplicación de las normas, la organización y el control de acciones para el desarrollo y bienestar materno-fetal. Sin embargo, para el cumplimiento de este objetivo, el hospital debe enfrentar cierta limitación de recursos materiales e insumos como hemoglobina y medicamentos, así como material de curación de calidad limitada.

Otro valioso ejemplo de conocimiento almacenado y socializado (CE), son las normas y guías técnicas para operar el sistema de Triage Obstétrico (semaforización de riesgo), cuya finalidad es priorizar e identificar situaciones potencialmente compr

ometedoras para la integridad del binomio madre-hijo, a partir de signos y síntomas relevantes en nueve factores para detectar una urgencia real, el procedimiento de recepción y la clasificación de acuerdo a la gravedad del padecimiento.

En el mismo sentido, el hospital cuenta con guías de práctica clínica en obstetricia (CE). Son documentos donde se plantean preguntas específicas y se organizan las mejores evidencias científicas disponibles para que, en forma de recomendaciones flexibles, sean utilizadas como herramienta de apoyo en la toma de decisiones clínicas. Por otro lado, también funcionan como malla curricular para la capacitación permanente del personal (CH). Aquí aplica la postura de Habersam y Piber (2010) en cuanto a que el capital intelectual es conocimiento gestionado, pero también conocimiento en sí.

Las guías de práctica clínica con que cuenta el nosocomio son: atención integral de preclamsia, detección y tratamiento de enfermedades hipertensivas en el embarazo, detección y tratamiento de las emergencias obstétricas, diagnóstico y manejo del parto pre término, diagnóstico y tratamiento de diabetes en el embarazo, diagnóstico y tratamiento de hemorragia obstétrica, prevención y manejo de hemorragia postparto, entre otras.

Para la detección oportuna, diagnóstico y tratamiento de trastornos del nacimiento, dichas guías están apegadas a la Norma Oficial Mexicana NOM 034-SSA3-2013, Regulación de los servicios de salud y Atención médica pre-hospitalaria, que incluye los principales defectos prevenibles o susceptibles de diagnóstico temprano.

Una de las acciones encaminadas a la detección oportuna de estas enfermedades es el tamiz metabólico neonatal, prueba de laboratorio practicada al recién nacido para detectar padecimientos de tipo congénito o metabólico. Este estudio se realiza entre el tercero y cuarto día del recién nacido, ya que, ante la detección y tratamiento oportunos, puede llegarse a la cura completa. Aunque este estudio pudiera 
realizarse en todas las unidades médicas del sector público de salud, este hospital recurre a un instituto externo ubicado en la Ciudad de México (CR) debido a la complejidad de los casos,

Los bancos de leche, como una estrategia más para disminuir la muerte neonatal, no entran en contradicción con el programa permanente de lactancia materna, ya que hacen posible el estudio y perfeccionamiento de técnicas de conservación de la leche materna (CE) para la alimentación del recién nacido ante la ausencia permanente de su madre o hasta que ella pueda amamantarlo adecuadamente.

Esta medida institucional tiene un efecto importante sobre la comunidad en donde ya opera (CR), porque la leche materna es un producto de gran valor que justifica la organización de un costoso dispositivo técnico con el fin de su preservación y dispensación (CE). El funcionamiento de banco de leche está sistematizado (procedimientos) en cinco rubros (CE): selección de las donantes, recolección de leche y traslado al banco de leche, procesamiento de la leche de banco, dispensación y administración de la leche de banco y seguridad de la leche de banco.

Para incrementar la cobertura del servicio especializado que representa el embarazo de alto riesgo, el sector salud ha construido una instalación estratégica de redes (CR) que incluye a hospitales en todo el Estado de México. Estas redes cuentan con un hospital ancla (hospitales generales o de segundo nivel) responsable del manejo de los casos de embarazos de alto riesgo provenientes de los hospitales de primer nivel (centros de salud, hospitales municipales y centros de atención primaria a la salud), en donde se realizan micro diagnósticos, se generan expedientes clínicos y, de ser el caso, se remite a la paciente al hospital de alta especialidad.

La labor de coordinación $(\mathrm{CH})$ de estas redes es de gran importancia, porque a través de ellas se integran controles prenatales, se evita saturar los hospitales de especialidad y alta especialidad y, al mismo tiempo, se brinda atención a todas las mujeres embarazadas que pertenezcan o no a una institución de salud pública.

Para iniciar este proceso, se utiliza la Hoja de Evaluación del Riesgo Materno, formato creado por especialistas de este hospital $(\mathrm{CH})$, con el cual se diagnostica la condición del embarazo de una mujer desde su ingreso en los hospitales de primer nivel. Este registro sirve de sustento para decidir sobre la canalización de los casos de embarazos según su gravedad.

Como puede verse, esta estrategia de redes es evidencia de capital relacional, pues su operación se reviste de relaciones internas y externas en y con el hospital de tercer nivel.

El capital relacional también se manifiesta en el vínculo entre paciente, familiares y personal de salud. Por ejemplo, un saludo cordial y la amabilidad abren espacio a la confianza y seguridad de quienes no se encuentran en un buen momento. La naturaleza de las relaciones clínico-administrativas internas se evidencia en el trabajo en equipo $(\mathrm{CH})$ para lograr la satisfacción del paciente, siendo resolutivos en sus patologías o en los ciclos de pláticas de lactancia. Aun así, parece que estas relaciones podrían mejorarse en la medida en que pacientes y familiares estén más informados, acción que combina interés y actitud y que se ha identificado como empoderamiento del paciente.

La atención a la situación crítica de un embarazo debe caracterizarse por la presencia del trabajo colaborativo de equipos multidisciplinarios $(\mathrm{CH})$ y la ausencia de relaciones internas $(\mathrm{CR})$ permeadas de celo profesional y falta de comunicación entre el personal de salud.

El banco de sangre coordina las acciones de captación, procesamiento, análisis, fraccionamiento, conservación y distribución de sangre humana, sus hemoderivados y células hematopoyéticas con fines te- 
rapéuticos, lo cual le da capacidad para apoyar a instancias similares del sector público, social y privado (CR), en la solución de problemas inmunohematológicos en la entidad (CE).

Este banco de alta especialidad también se vincula con el Centro Estatal de la Transfusión Sanguínea para el suministro de insumos, equipo y material, así como la vigilancia al cumplimiento de la normatividad aplicable para su operación y las medidas de bioseguridad para protección del personal (CE, $\mathrm{CR}$ y CH).

El área de trabajo social es el enlace entre el paciente y el equipo de salud (CR), lo cual se traduce en capital relacional. Por ejemplo, implementa acciones orientadas a resolver los problemas sociales que influyen en la prevención, control y rehabilitación de los pacientes que se atienden en el hospital; da apoyo y orientación a los pacientes que reciban asistencia médica de urgencia; realiza estudios socioeconómicos para determinar la clasificación de pago por los servicios recibidos.

A modo de relaciones externas (CR), el hospital materno perinatal lleva a cabo acciones de participación ciudadana, las cuales van más allá de las fronteras del hospital y permean en las redes hospitalarias, coordinaciones y jurisdicciones sanitarias. Ejemplo de dichas acciones son: (a) el buzón de quejas, sugerencias y felicitaciones, donde el actor principal es el aval ciudadano; (b) la formación de grupos de captación, recolección y capacitación de donadoras de leche materna, que son mujeres mexiquenses organizadas para que en su domicilio, colonia o municipio puedan difundir las técnicas de preservación de leche materna, así como servir de punto de referencia para nuevas donadoras.

Lo anterior bien puede ser considerado como innovación organizativa, la cual según Westbrook y Schultz (2000) implica dirigir recursos intangibles, como tiempo y experiencia $(\mathrm{CH})$, hacia la construcción de alianzas entre la comunidad y el personal de salud (CR), para fortalecer la capacidad de participación de los habitantes en este proceso de mejoramiento de la salud comunitaria (relaciones externas). Así, equipos multidisciplinarios (relaciones internas) pueden contribuir al cumplimiento de metas establecidas en la política de salud, específicamente en cuanto a la permanente reducción de tasa de muerte materna.

\subsection{Codependencia del capital intelectual y los recursos tangibles}

El capital intelectual no sustituye a los recursos intangibles, pero sí los potencializa, es decir, la gestión del capital intelectual puede ser un catalizador en el desempeño de las instituciones hospitalarias y, por lo tanto, en la atención de salud a la población objetivo. De acuerdo a Evans, et al. (2015) en algunos casos el capital intelectual debe estar acompañado de recursos tangibles para contribuir a la generación de valor y a un desempeño superior. Específicamente en este hospital de alta especialidad, se han detectado algunos ejemplos.

Cuando no se sistematiza la información, los datos y la evidencia médica en materia de políticas de salud, administración hospitalaria, prestación de asistencia sanitaria y experiencias documentadas de casos clínicos, se pudiera correr el riesgo de no detonar el aprendizaje organizacional, ni recurrir a la evidencia empírica para la toma de decisiones que lleve a disminuir la tasa de mortandad materna y neonatal. De aquí la importancia de los sistemas de información y tecnología, los cuales no se podrían entender sin la presencia de recursos tangibles. 
La permanente actualización del personal de salud (médico y paramédico) está vinculada a la normatividad y tecnología hospitalarias. Tal es el caso de la enfermera especializada en reanimación neonatal, figura demandada en cada turno y en cada servicio como parte del equipo de emergencias (Código Mater).

Hasta hace tres décadas, la única solución para las parejas infértiles era la adopción. Este hospital cuenta con equipo y tecnología de vanguardia para tratamientos de reproducción asistida de baja y alta complejidad como inseminación artificial y fertilización in vitro. Brinda atención a mujeres entre 18 y 36 años que no han logrado concebir un embarazo después de un año de buscarlo sin protección anticonceptiva. Como manifestación del capital estructural, los criterios de admisión y los requisitos de ingreso, están debidamente definidos y son dominados por los responsables del área.

En este hospital se vive la experiencia de transitar del expediente clínico físico al expediente clínico electrónico (recurso intangible). Sin embargo, para implementar y operar el respectivo sistema se recurre a servicios especializados de una empresa externa, lo que encierra un compromiso financiero, el cual debe mantenerse para garantizar su permanente disposición y no truncar este logro.

Como lo afirman Hermans y Kauranen (2005), la imagen y reputación organizacionales son resultado de la integración de recursos tangibles e intangibles. En el caso que nos ocupa, su fuerte compromiso social con la comunidad económicamente vulnerable le demanda mayor inversión (tangible e intangible).

Finalmente, y a partir de la relación positiva entre la participación en la elaboración de planes de trabajo y propuestas de solución con el compromiso personal hacia la organización (Meyer y Allen, 1991), los participantes en este estudio (médicos y paramédicos), consideran que para optimizar el capital intelectual del hospital podrían detonarse las competencias $(\mathrm{CH})$ al hacer una revisión de asignación de puestos y responsabilidades, así como un diagnóstico del clima y cultura organizacionales.

Tan importante como lo anterior, es la eficiencia en los servicios hospitalarios (CE) soportada en el trabajo conjunto multidisciplinario, cuyo aprendizaje puede hacerse extensivo a otros hospitales (CR), específicamente en lo referente al Código Mater. Éstas y otras propuestas deben estar encaminadas a disminuir el ya referido impacto social de la muerte materna, objetivo que se logra en colaboración y con responsabilidad (intangibles) de los involucrados internos y externos, pero también con la disponibilidad de recursos tangibles como presupuesto y tecnología de punta.

\section{Conclusión}

El capital intelectual en la práctica organizacional vive todavía una etapa embrionaria de desarrollo, aunque las publicaciones científicas crecen aceleradamente.

Ante la ausencia de recomendaciones comprensibles y explícitas de la manera en que el capital humano, el capital estructural y el capital relacional están anidados y actúan con recursos tangibles para favorecer el desempeño superior de las instituciones de salud, este caso de estudio en un hospital materno perinatal de alta especialidad logra reportar con una lente matizada, las manifestaciones y ejemplos de capital intelectual que pueden contribuir a la misión de disminuir la razón de muerte materna en mujeres mexiquenses económica y socialmente vulnerables.

En este hospital ya existen mecanismos para cultivar el capital humano, aunque también la necesidad de más capacitación y mejor preparación del personal, tanto en áreas de la salud como en administración de hospitales y gestión de la calidad, con la finalidad de brindar bienestar a los pacientes. 
El soporte que el capital estructural genera a las actividades cotidianas del nosocomio, se traduce en la construcción de comités y la normatividad actualizada y pertinente, tal como: planes de trabajo, guías de práctica clínica, certificaciones, banco de leche y banco de sangre, entre otros.

Parecería que, entonces, el capital relacional tiene su detonante tanto en las relaciones externas como en las internas. Aquéllas están entretejidas en las redes de hospitales, en el enlace que trabajo social construye con las comunidades y en las acciones de participación ciudadana. Las relaciones internas están en el vínculo paciente-familiares y personal de salud.

En esencia, este hospital de alta especialidad puede incluir de manera manifiesta a los recursos intangibles, específicamente al capital intelectual, en su modelo de gestión hospitalaria.

El escenario aquí presentado está acentuado sobre el quehacer hospitalario para disminuir, desde un enfoque de eficiencia médica, la muerte materna. Sin embargo, parece importante reforzar su contribución inter-organizacional para que, a partir de tangibles e intangibles, se ataquen las causas raíz, como son la condición psicosocial de las mujeres de escasos recursos económicos y educativos, en donde a veces la pareja las abandona y se hacen parte de la segregación social. En otras palabras, comprender el contexto en el que se desarrolla el embarazo y ocurre la muerte, contribuiría a construir soluciones más acordes con las necesidades de la mujer.

Para continuar explorando sobre el capital intelectual en hospitales, puede optarse por el enfoque comparativo mediante el estudio de casos centrado en la identificación y descomposición del capital intelectual en diversas organizaciones sanitarias y su influencia en el rendimiento. $O$ bien, realizar estudios cuyo alcance se centre en departamentos o iniciativas particulares dentro o entre organizaciones, además de la incursión en estudios longitudinales versus las políticas estatales y nacionales de salud pública, con especial atención a la prevención.

Aunque la literatura sobre capital intelectual en instancias del cuidado a la salud está más enfocada a nivel organizacional, el capital intelectual también es relevante y está presente en redes inter-organizacionales y en el desempeño de todo un sistema de salud. En esencia, el avance del estudio del capital intelectual demanda la aplicación de mejores prácticas en el diseño de investigación y el reporte de métodos y resultados.

\section{Referencias}

Ahmad, Abdel-Azis, Shawqi Naji y Nick Bontis. 2010. Intellectual capital and business performance in the pharmaceutical sector of Jordan. Management Decision. 18(1):105-131.

Amos, Elizabeth y Bart Weathington. 2008. An analysis of the relation between employee-organization value congruence and employee attitudes. The Journal of Psychology, 142(6): 615-631.

Aristizábal, Juan, Germán Gómez, Juan Lopera, Laura Orrego, Carlos Restrepo, Germán Monsalve, Martín Gómez, Nury Socha y Mauricio Vasco. 2005. Paciente obstétrica de alto riesgo: ¿dónde debe realizarse su cuidado periparto? Revista Colombiana de Obstetricia y Ginecología, 56(2): 166-175.

Barney, Jay. 1991. Firm resources and sustained competitive advantage. Journal of Management, 17(1): 99-120

Bontis, Nick, Mary Crossan y John Hulland. 2002. Managing and organizational learning system by 
aligning stock and flows. Journal of Management Studies, 39(4): 437-469.

Bontis, Nick. 1999. Managing organizational knowledge by diagnosing intellectual capital: Framing and advancing the state of the field. International Journal of Technology Management, 18(5/6/7/8): 433-462. Booker, Lorne, Nick Bontis y Alexander Serenko. 2008. The relevance of knowledge management and intellectual capital. Knowledge and Process Management, 15(4): 235-246.

Bradley, Keith. 1997. Intellectual capital and the new wealth of nations. Business Strategy Review, 8(1): 53-62.

Creswell, John W. 2009. Research design. Qualitative, Quantitative, and Mixed Methods Approaches. Londres: SAGE Publications

Donoso, Bernardita y Enrique Oyarzún. 2012. Embarazo de alto riesgo. MedWave, 12(5). DOI: http:// doi.org/10.5867/medwave.2012.05.5429

Dumay, John. 2012. Grand theories as barriers to using IC concepts. Journal of Intellectual Capital, 13(1): 4-15. DOI: http://doi.org/10.1108/14691931211196187

Eaton, Adrienne y Thomas Nocerino. 2000. The effectiveness of health and safety committees: Results of a survey of public-sector workplaces. Industrial Relations, 39(2): 265-290.

Evans, Jenna, Adalsteinn Brown y Ross Beker. 2015. Intellectual capital in the healthcare sector: a systematic review and critique of the literature. BMC Health Services Research, 15:556-569.

Habersam, Michael y Martin Piber. 2010. Exploring intellectual capital in hospitals: two qualitative case studies in Italy and Austria. Journal European Accounting Review, 12(4): 753-779.

Hermans, Raine e Ilkka Kauranen. 2005. Value creation potential of intellectual capital in biotechnology - empirical evidence from Finland. R\&D Management, 35(2): 171-185.

Instituto de Salud del Estado de México. 2017. Portal institucional. http://www.salud.edomexico.gob.mx (3 de febrero de 2018).

Jang, Seung Hoon. 2013. The offensive framework of resource based view (RBV): Inhibiting others from pursuing their own values. Journal of Management and Strategy, 4(1): 62-69. DOI: http://doi.org/10.5430/ jms.v4n1p62

Kannan, Gopika y Wilfried Aulber. 2004. Intellectual capital: Measurement effectiveness. Journal of Intellectual Capital, 5(3): 389-413. DOI: http://doi.org/10.1108/14691930410550363

Karam, Miguel, Patricia Bustamante, Martha Campuzano y Ángela Camarena. 2007. Aspectos sociales de la mortalidad materna. Estudio de caso en el Estado de México. Medicina Social, 2(4): 2005-2011.

Kong, Eric y Daniel Prior. 2008. An intellectual capital perspective of competitive advantage in nonprofit organisations. International Journal of Non-profit and Voluntary Sector Marketing, 13(2): 119-128. Kong, Eric. 2008. The development of strategic management in the non-profit context: Intellectual capital in social service non-profit organizations. International Journal of Management Reviewers, 10(3): 281299.

Lev, Baruch y Stefano Zambon. 2003. Intangibles and intellectual: capital an introduction to a special issue. European Accounting Review, 12(4): 597-603.

Lozano, Leidy, Alix Bohórquez y Gloria Zambrano. 2016. Implicaciones familiares y sociales de la muerte materna. Revista Univ Salud, 18(2): 364-372.

Luthans, Fred. 1989. Organizational behavior. Nueva York: McGraw Hill. 
- Revista de Ciencias Sociales y Humanidades. ISSN-P: 0188-9834 ISSN-E:2395-8669.

Meyer, John y Natalie Allen. 1991. A three-component conceptualization of organizational commitment. Human Resources Management Review, 1(1): 61-89.

Remenyie, Dan. 2012. Case study research. The quick guide. Londres: ACPI.

Roos, Johan, Göran Roos, Nicola Dragonetti y Leif Edvinsson. 2001. Capital intelectual. El valor intangible de la empresa. España: Paidós.

Stewart, Thomas. 1997. La nueva riqueza de las organizaciones: el capital intelectual. Buenos Aires: Garnica. Tzu-Ju, Ann. 2011. Resource fit in inter-firm partnership: intellectual capital perspective. Journal of Intellectual Capital, 12(1): 20-42. DOI: http://doi.org/10.1108/14691931111097908

Westbrook, Linda y Phyllis Schultz. 2000. Form theory to practice: Community health nursing in a public health neighborhood team. Advances in Nursing Science, 23(2): 50-61.

Yin, Robert. 2013. Case study research: Design and methods. California: SAGE. 